GENERALIZATIONS OF COMPLEX ANALYSIS

BANACH CENTER PUBLICATIONS, VOLUME 37

INSTITUTE OF MATHEMATICS

POLISH ACADEMY OF SCIENCES

WARSZAWA 1996

\title{
HURWITZ ANALYSIS: BASIC CONCEPTS AND CONNECTION WITH CLIFFORD ANALYSIS
}

\author{
ENRIQUE RAMÍREZ DE ARELLANO and NIKOLAI VASILEVSKI \\ Departamento de Matemáticas \\ Centro de Investigación y de Estudios Avanzados \\ del Instituto Politécnico Nacional \\ 07000 México, D.F., México \\ MICHAEL SHAPIRO \\ Departamento de Matemáticas \\ E.S.F.M., Instituto Politecnico Nacional \\ U.P.A.L.M., Edificio No. 9, Zacatenco \\ 07000 México 14, D.F., México
}

Introduction. The Hurwitz problem stated by him in 1898 [H1] and its further development [H2] were the motivation to introduce and to study the so-called Hurwitz pairs (see [Sh], [SY] for an extensive literature).

In our works [RSV1], [RSV2] the precise relation between Hurwitz pairs and Clifford algebras has been established. Two canonical algorithms have been described for constructing an irreducible representation of a certain Clifford algebra for a given Hurwitz pair and, conversely, for constructing all possible Hurwitz pairs from a given Clifford algebra and its irreducible representation.

All this has been inspired by our wish to develop the so-called Hurwitz analysis initiated in [KR1], [KR2].

The first Section of the present paper is purely algebraic. Besides the review of main results from [RSV1], [RSV2], it contains some essential supplements. The principal one is how "to translate" any formula of the type $a=\sum_{i} b_{i} \cdot c_{i}$ in a Clifford algebra into the language of the multiplication in the Hurwitz pair.

In Section 2 we introduce two types of generalized Cauchy-Riemann operators and show that all main formulas for the functions from the kernels of these operators, can be

1991 Mathematics Subject Classification: Primary 46C20; Secondary 32G81.

Partially supported by CONACYT project 1821-E9211.

The paper is in final form and no version of it will be published elsewhere. 
obtained in the traditional way. And thus, we get two types of analysis (i.e., the analogs of the function theory in one complex variable) associated to a fixed Hurwitz pair.

Finally, in Section 3, we show that the proper way to construct both types of Hurwitz analysis is to derive it from Clifford analysis. The derivation is being done by means of the "translation" described in Section 1.

\section{Preliminaries}

1.1. Let $S$ be a $(p+1)$-dimensional real vector space with basis $\left\{\epsilon_{\alpha}\right\}, \alpha \in\{0\} \cup \mathbb{N}_{p}$, $\mathbb{N}_{p}:=\{1,2, \ldots, p\}$, and let the $\mathbb{R}$-bilinear form

$$
(\cdot, \cdot)_{S}: S \times S \rightarrow \mathbb{R}
$$

be defined by the following metric matrix

$$
\eta:=\left[\eta_{\alpha \beta}\right]:=\left[\left(\epsilon_{\alpha}, \epsilon_{\beta}\right)_{S}\right]=\operatorname{diag} \frac{(1, \ldots, 1}{\mathrm{r}+1} \frac{-1, \ldots,-1)}{\mathrm{s}}
$$

where $p=r+s$.

Introduce also the $n$-dimensional real vector space $V$ with basis $\left\{e_{j}, j \in \mathbb{N}_{n}\right\}$, provided with an $\mathbb{R}$-bilinear form

$$
(\cdot, \cdot)_{V}: V \times V \rightarrow \mathbb{R}
$$

which is defined by the following nonsingular metric matrix

$$
\kappa:=\left[\kappa_{k j}\right]:=\left[\left(e_{k}, e_{j}\right)_{V}\right] .
$$

We assume also that the form $(\cdot, \cdot)_{V}$ in $V$ is either symmetric: $\kappa=\kappa^{t}$, or antisymmetric: $\kappa=-\kappa^{t}$, where " $t$ " means transposition.

Let

$$
\circ: S \times V \rightarrow V
$$

be an $\mathbb{R}$-bilinear mapping. We call it (see, for instance $[\mathrm{LR}]$ ) a Hurwitz multiplication (of elements from $V$ by elements of $S$ on the left-hand side) if the following axioms are fulfilled:

H.1. for all $\{f, g\} \subset V$ and all $a \in S$

$$
(a, a)_{S}(f, g)_{V}=(a \circ f, a \circ g)_{V}
$$

H.2. there exists the unit element $\epsilon \in S$ with respect to the mapping " $\circ$ ", i.e. for all $f \in V$

$$
\epsilon \circ f=f
$$

H.3. the mapping " $\circ "$ does not leave invariant any proper subspace of $V$. The set $(S, V, \circ)$ is called a Hurwitz pair.

1.2. Introduce the $\mathbb{R}$-linear isomorphisms

$$
\nu_{S}: S \rightarrow \mathbb{R}^{p+1}
$$

and

$$
\nu_{V}: V \rightarrow \mathbb{R}^{n}
$$


by the rule

$$
\nu_{S}: a=\sum_{\alpha=0}^{p} a_{\alpha} \epsilon_{\alpha} \mapsto \tilde{a}=\left(\begin{array}{c}
a_{0} \\
a_{1} \\
\vdots \\
a_{p}
\end{array}\right) \in \mathbb{R}^{p+1}
$$

and

$$
\nu_{S}: f=\sum_{j=1}^{n} f_{j} e_{j} \mapsto \tilde{f}=\left(\begin{array}{c}
f_{0} \\
f_{1} \\
\vdots \\
f_{n}
\end{array}\right) \in \mathbb{R}^{n} .
$$

For each basis elements $\epsilon_{\alpha} \in S$ and $e_{j} \in V$ we have $\epsilon_{\alpha} \circ e_{j} \in V$. Thus for some real constants $c_{\alpha j}^{k}$ :

$$
\epsilon_{\alpha} \circ e_{j}=\sum_{k=1}^{n} c_{\alpha j}^{k} e_{k}
$$

and for every $f=\sum_{j=1}^{n} f_{j} e_{j} \in V$ :

$$
\epsilon_{\alpha} \circ f=\sum_{k=1}^{n}\left(\sum_{j=1}^{n} c_{\alpha j}^{k} f_{j}\right) e_{k} .
$$

Applying the isomorphism $\nu_{v}$ to the both sides of this equality we obtain

$$
\nu_{V}\left(\epsilon_{\alpha} \circ f\right)=C_{\alpha} \cdot \nu_{V}(f),
$$

where $C_{\alpha}:=\left[c_{\alpha j}^{k}\right]_{j, k=1}^{n}$.

Thus, each element $\epsilon_{\alpha} \in S$ determines uniquely the matrixx $C_{\alpha}$ (and vice versa), and the following diagram

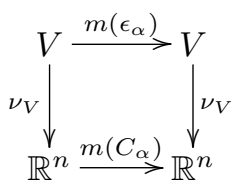

is commutative. Here $m\left(\epsilon_{\alpha}\right): f \mapsto \epsilon_{\alpha} \circ f$ and $m\left(C_{\alpha}\right): \tilde{f} \mapsto C_{\alpha} \cdot \tilde{f}$.

1.3. Without loss of generality we may assume that the unit element (三 identity $\epsilon \in S)$ coincides with $\epsilon_{0}$.

Under the assumption we have (see $[\mathrm{RSV} 1])$ : for each $\{\alpha, \beta\} \subset \mathbb{N}_{p}$

$$
C_{\alpha} \cdot C_{\beta}+C_{\beta} \cdot C_{\alpha}=-2 \eta_{\alpha \beta} I_{n} .
$$

1.4. Given integers $r, s$, with $p=r+s$, denote by $C l_{r, s}$ the real Clifford algebra with the identity $i_{0}$ and generated by the elements $\left\{i_{\alpha}: \alpha \in \mathbb{N}_{p}\right\}$ which satisfy only the conditions

$$
i_{\alpha} \cdot i_{\beta}+i_{\beta} \cdot i_{\alpha}=-2 \eta_{\alpha \beta} i_{0}
$$

where

$$
\eta_{\alpha \beta}= \begin{cases}0, & \alpha \neq \beta \\ +1, & \alpha=\beta \in \mathbb{N}_{r} \\ -1, & \alpha=\beta \in \mathbb{N}_{p} \backslash \mathbb{N}_{r}\end{cases}
$$


1.5. TheOREM ([RSV1]). Let $(S, V, \circ)$ be a Hurwitz pair with $\epsilon=\epsilon_{0}$. Then there exists an $n$-dimensional irreducible representation

$$
\pi_{n}: C l_{r, s} \rightarrow \mathbb{R}(n)
$$

of the Clifford algebra $C l_{r, s}$, and this representation is generated by the following mapping:

$$
\pi_{n}: i_{\alpha} \mapsto C_{\alpha},
$$

where $\mathbb{R}(n)$ denotes the algebra of all real $n \times n$ matrices and $C_{\alpha}$ is as in 1.2 .

1.6. Consider a fixed Hurwitz pair $(S, V, \circ)$ with the unit element $\epsilon=\epsilon_{0}$. Each element $a \in S$ generates in a natural way the operator of "Hurwitz multiplication by $a$ " acting on $V$ by the rule

$$
f \in V \mapsto a \circ f \in V .
$$

Denote this operator by $m(a)$. It is clear that for $a=\sum_{\alpha=0}^{p} a_{\alpha} \epsilon_{\alpha}$

$$
m(a)=\sum_{\alpha=0}^{p} a_{\alpha} m\left(\epsilon_{\alpha}\right)
$$

where $m\left(\epsilon_{0}\right)=I$, the identity operator on $V$.

The mapping

$$
\mu: a \mapsto m(a) \in \operatorname{Hom}(V, V)
$$

gives a linear isomorphism between the space $S$ and some linear subspace of $\operatorname{Hom}(V, V)$.

Denote by $\operatorname{Alg}(S, V, \circ)$ the algebra generated by all operators $m(a)$ acting on $V$. We have obviously

$$
\mu(S) \subset \operatorname{Alg}(S, V, \circ) \subset \operatorname{Hom}(V, V) \cong \mathbb{R}(n),
$$

and in general both inclusions are proper.

1.7. Theorem ([RSV1]). The algebra $\operatorname{Alg}(S, V, \circ)$ is isomorphic to the algebra $\pi_{n}\left(C_{r, s}\right)$ and this isomorphism is generated by the following mapping

$$
m\left(\epsilon_{\alpha}\right) \mapsto m\left(C_{\alpha}\right), \quad \alpha \in\{0\} \cup \mathbb{N}_{p} .
$$

All above said can be found in [RSV1], [RSV2], but to construct the corresponding function theory we need to complement the algebraic part of those works with some new results.

1.8. The algebra $\operatorname{Alg}(S, V, \circ)$ allows us to introduce a multiplication on elements of $S$ in such a way that the algebra $\tilde{S}$ generated by this multiplication becomes isomorphic to $\operatorname{Alg}(S, V, \circ)$. The corresponding isomorphism will be an extension of the linear mapping $\mu$ from $S$ onto the algebra $\tilde{S}$.

Denote by $\check{S}$ the free algebra generated by the elements of $S$ and let

$$
\check{\mu}: \check{S} \rightarrow \operatorname{Alg}(S, V, \circ)
$$

be the real algebra homomorphism which is generated by the following mapping of the generators $a \in S$ of the algebra $\check{S}$ :

$$
\check{\mu}: a \in S \mapsto \check{\mu}(a):=\mu(a)=m(a) \in \operatorname{Hom}(V, V),
$$


i.e. the mapping $\check{\mu}$ is an extension (up to a real algebra homomorphism) of the mapping

$$
\mu: S \rightarrow \operatorname{Alg}(S, V, \circ) .
$$

Now introduce the algebra $\tilde{S}:=\check{S} / \operatorname{ker} \check{\mu}$ and the mapping

$$
\tilde{\mu}: \tilde{S} \rightarrow \operatorname{Alg}(S, V, \circ)
$$

which is defined by the rule

$$
\tilde{\mu}:[\check{a}] \mapsto \check{\mu}(\check{a})
$$

where $[\check{a}]:=\check{a}+\operatorname{ker} \check{\mu}$ for $\check{a} \in \check{S}$.

We will be denote by "*" the multiplication symbol in $\tilde{S}$.

The described procedure provides a natural imbedding of $S$ into $\tilde{S}$. Identifying, as usual, $S$ and its image under this imbedding, we can say now that $S$ is a subset (and a linear subspace) of the algebra $\tilde{S}$. It is clear that

$$
\tilde{\mu} \mid S=\mu .
$$

The mapping $\tilde{\mu}$ gives now a real algebra isomorphism of $\tilde{S}$ onto $\operatorname{Alg}(S, V, \circ) \subset \operatorname{Hom}(V, V)$, and thus also a representation of the algebra $\tilde{S}$ on the space $V$.

Moreover, we have a well-defined extension (from $S \times V$ onto $\tilde{S} \times V$ ) of the Hurwitz multiplication, also denoted by "o", as follows:

$$
\text { for each } s \in \tilde{S} \text { and } f \in V \quad s \circ f:=\tilde{\mu}(S)(f) \in V \text {. }
$$

1.9. Remark. For any elements $s_{1}, s_{2} \in \tilde{S}$ and $f \in V$ the following "associativity law" is true

$$
\left(s_{1} * s_{2}\right) \circ f=s_{1} \circ\left(s_{2} \circ f\right),
$$

and thus we can write $s_{1} * s_{2} f:=\left(s_{1} * s_{2}\right) \circ f=s_{1} \circ\left(s_{2} \circ f\right)$.

1.10. THEOREM. Let $(S, V, \circ)$ be a Hurwitz pair and $\tilde{S}$ the algebra described in 1.8. Then there exists a natural number $k$ such that $\tilde{S}$, as a linear space, is isomorphic to a finite direct sum of $k$ copies of $V$ :

$$
\tilde{S} \cong V \oplus \ldots \oplus V \quad(k \text { times }) .
$$

Proof. Denote by

$$
\rho: \tilde{S} \rightarrow \operatorname{Hom}(\tilde{S}, \tilde{S})
$$

the left regular representation of the algebra $\tilde{S}$ which is defined by the rule

where $m(s): x \in \tilde{S} \mapsto s * x \in \tilde{S}$.

$$
\rho: s \in \tilde{S} \mapsto m(s) \in \operatorname{Hom}(\tilde{S}, \tilde{S}),
$$

The mapping $\rho$ gives a real algebra isomorphism between $\tilde{S}$ and its image $\rho(\tilde{S}) \subset$ $\operatorname{Hom}(\tilde{S}, \tilde{S})$. Another natural representation of the algebra $\tilde{S}$ is given on the space $V$ by the mapping (1.1)

$$
\tilde{\mu}: \tilde{S} \rightarrow \operatorname{Alg}(S, V, \circ) \subset \operatorname{Hom}(V, V)
$$

where, for each $s \in \tilde{S}$

$$
\tilde{\mu}(s)=m(s): f \in V \mapsto s \circ f \in V .
$$

The mapping $\tilde{\mu}$ is also a real algebra isomorphism between $\tilde{S}$ and its image $\operatorname{Alg}(S, V, \circ)$. 
Thus we have two representations of the irreducible finite-dimensional algebra $\tilde{S}$ on the spaces $\tilde{S}$ and $V$ with (generally speaking) different dimensions:

$$
\operatorname{dim} V \leq \operatorname{dim} \tilde{S}
$$

and their images are isomorphic. This proves the assertion.

1.11. Corollary. The representation $\rho$ in the proof of Theorem 1.10 is isomorphic to a multiple of the representation $\tilde{\mu}$ :

$$
\rho \cong \tilde{\mu} \oplus \ldots \oplus \tilde{\mu} \quad(k \text { times }) .
$$

This means that there exists an invertible element $\gamma \in \operatorname{Hom}(\tilde{S}, \tilde{S})$ such that

$$
\gamma(\tilde{S})=V \oplus \ldots \oplus V \quad(k \text { times })
$$

and that for all $s \in \tilde{S}$

$$
\gamma \rho(s) \gamma^{-1}=\tilde{\mu}(s) \oplus \ldots \oplus \tilde{\mu}(s) \quad(k \text { times }) .
$$

1.12. Denote by $P$ the projection onto the first summand in the decomposition

$$
\gamma(\tilde{S})=V \oplus \ldots \oplus V \quad(k \text { times }) .
$$

Then for each $s \in \tilde{S}$

$$
\tilde{\mu}(s)=\left.\operatorname{P\gamma } \rho(s)^{-1}\right|_{V} .
$$

Note that the projection $P$ commutes with all elements of the form $\gamma \rho(s) \gamma^{-1}$.

Denote by $v$ the isomorphism

$$
\nu: \gamma \operatorname{Im} \rho \gamma^{-1} \rightarrow \operatorname{Alg}(S, V, \circ),
$$

where $\nu:\left.\gamma \rho(s) \gamma^{-1} \mapsto P \gamma \rho(s) \gamma^{-1}\right|_{V}$. Then we have the following commutative diagram of algebras:

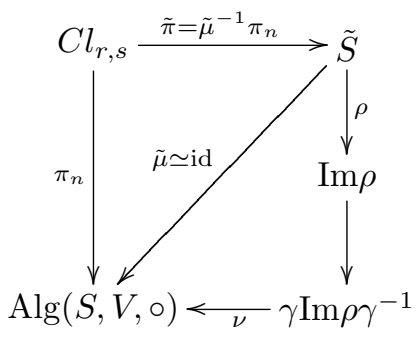

1.13. Let $\left\{a, b_{j}, c_{i}\right\}$ be a finite subset of elements from the Clifford algebra $C l_{r, s}$ and let

$$
a=\sum_{i} b_{i} \cdot c_{i} .
$$

Application of the above constructed homomorphisms and isomorphisms gives us the "translation" of this equality into $\tilde{S}$ and $V$ respectively:

$$
\begin{gathered}
\text { in } \tilde{S}: \tilde{\pi}(a)=\sum_{i} \tilde{\pi}\left(b_{i}\right) * \tilde{\pi}\left(c_{i}\right) \\
\text { in } V: \operatorname{P\gamma } \tilde{\pi}(a)=\sum_{i} \operatorname{P\gamma }\left(\tilde{\pi}\left(b_{i}\right) * \tilde{\pi}\left(c_{i}\right)\right)=\sum_{i} \operatorname{P\gamma } \rho\left(\tilde{\pi}\left(b_{i}\right)\right) \cdot \tilde{\pi}\left(c_{i}\right)=
\end{gathered}
$$




$$
\begin{gathered}
=\sum_{i} P \gamma \rho\left(\tilde{\pi}\left(b_{i}\right)\right) \gamma^{-1} \cdot \gamma \tilde{\pi}\left(c_{i}\right)=\left.\sum_{i} P \gamma \rho\left(\tilde{\pi}\left(b_{i}\right)\right) \gamma^{-1}\right|_{V} \cdot P \gamma \tilde{\pi}\left(c_{i}\right)= \\
=\sum_{i} \tilde{\mu}\left(\tilde{\pi}\left(b_{i}\right)\right) \cdot P \gamma \tilde{\pi}\left(c_{i}\right)=\sum_{i} \pi\left(b_{i}\right) \circ P \gamma \tilde{\pi}\left(c_{i}\right)
\end{gathered}
$$

or, finally,

$$
P \gamma \tilde{\pi}(a)=\sum_{i} \pi\left(b_{i}\right) \circ P \gamma \tilde{\pi}\left(c_{i}\right)
$$

1.14. Analogously, for a finite subset $\left\{s, x_{i}, y_{j}\right\}$ of the algebra $\tilde{S}$, if

$$
s=\sum_{i} x_{i} * y_{i}
$$

then we have the corresponding equality in $V$ :

$$
P \gamma(s)=\sum_{i} x_{i} \circ P \gamma\left(y_{i}\right) .
$$

\section{Hurwitz analysis: exact formulation and construction in the traditional way}

2.1. Given a Hurwitz pair $(S, V, \circ)$ (with no restrictions on $\eta$ ), denote by $\ell$ any integer with the condition $2 \leq \ell \leq p$, and let $\Omega$ be a domain in $\mathbb{R}^{\ell+1}$. For any set of vectors $\left(\psi^{0}, \psi^{1}, \ldots, \psi^{\ell}\right)=: \psi \in S^{\ell+1}$ we can write the formal expression

$$
\sum_{\alpha=0}^{\ell} \psi^{\alpha} \circ \frac{\partial}{\partial x_{\alpha}}
$$

where $\frac{\partial}{\partial x_{\alpha}}$ denotes the operation of the usual partial derivation of a given $(\tilde{S}$-valued or $V$-valued) function defined in $\Omega$. Depending on the type of function the expression (2.1) allows to introduce two kinds of operators.

2.2. For an arbitrary function $f \in \mathcal{C}^{1}(\Omega, V)$ define the operator $\mathcal{D}_{\psi}$ by the rule

$$
\mathcal{D}_{\psi}[f]:=\sum_{\alpha=0}^{\ell} \psi^{\alpha} \circ \frac{\partial}{\partial x_{\alpha}}[f]:=\sum_{\alpha}^{\ell} \psi^{\alpha} \circ \frac{\partial f}{\partial x_{\alpha}} .
$$

The operator $\mathcal{D}_{\psi}$ becomes later the Cauchy-Riemann operator in the analysis of $V$-valued functions. It is necessary to emphasize strongly that just here we see the consequence of the Hurwitz multiplication asymmetry: in contrast with the usual hyperholomorphic setting we can introduce the left operator only, not the left and the right ones (compare with what will be done below).

2.3. Consider an arbitrary $g \in \mathcal{C}^{1}(\Omega, \tilde{S})$. Introduce the left ${ }^{\psi} \mathfrak{D}$ and the right $\mathfrak{D}^{\psi}$ analogs of the operator $\mathcal{D}_{\psi}$ by the rules:

$$
\psi \mathfrak{D}[g]:=\sum_{\alpha=0}^{\ell} \psi^{\alpha} * \frac{\partial}{\partial x_{\alpha}}[g]:=\sum_{\alpha}^{\ell} \psi^{\alpha} * \frac{\partial g}{\partial x_{\alpha}}
$$


and

$$
\mathfrak{D}^{\psi}[g]:=\sum_{\alpha=0}^{\ell} \psi^{\alpha} * \frac{\partial}{\partial x_{\alpha}} * m\left(\psi^{\alpha}\right)[g]:=\sum_{\alpha}^{\ell} \psi^{\alpha} \circ \frac{\partial g}{\partial x_{\alpha}} * \psi^{\alpha},
$$

where $m$ is the map defined in 1.6.

2.4. Using the notion of the "natural conjugation" on $S$ we introduce the "conjugate" operators $\mathcal{D}_{\psi}^{\sharp},{ }^{\psi} \mathfrak{D}^{\sharp}$ and $\mathfrak{D}^{\psi \sharp}$ :

$$
\begin{aligned}
\mathcal{D}_{\psi}^{\sharp}[f]: & =\sum_{\alpha=0}^{\ell} \psi^{\alpha \sharp} \circ \frac{\partial}{\partial x_{\alpha}}[f], \\
\psi_{\mathfrak{D}^{\sharp}}^{\sharp}[g]: & =\sum_{\alpha=0}^{\ell} \psi^{\alpha \sharp} * \frac{\partial}{\partial x_{\alpha}}[g], \\
\mathfrak{D}_{\psi \sharp}[g]: & =\sum_{\alpha=0}^{\ell} \frac{\partial}{\partial x_{\alpha}}[g] * \psi^{\alpha \sharp},
\end{aligned}
$$

where "\#" is a linear mapping on $S$ defined on the basis elements $\epsilon_{k}$ by the rule

$$
\epsilon_{k}^{\sharp}=-\epsilon_{k}, \quad k \in \mathbb{N}_{p} .
$$

2.5. Remark. To develop the corresponding function theory it is necessary to be able to multiply the above defined operators.

The following pecularities arise from the asymmetry of the Hurwitz multiplication. We have sets of $S$-valued functions, of $V$-valued functions, of $\tilde{S}$-valued operators (that is, differential operators with coefficients from $\tilde{S}$ ), etc. We can "multiply" $V$-valued functions on the left-hand side by $\tilde{S}$-valued operators obtaining $V$-valued functions: ${ }^{\psi} \mathfrak{D} \circ f:={ }^{\psi} \mathfrak{D}[f]$.

We can "multiply" various $\tilde{S}$-valued operators on both sides resulting in $\tilde{S}$-valued operators: $\psi \mathfrak{D} *^{\psi} \mathfrak{D}^{\sharp}:=\psi \mathfrak{D} \cdot \psi^{\sharp} \mathfrak{D}^{\sharp}$ with the " . denoting the usual operator product. We should take into account that, according to the definition of $\psi \mathfrak{D}$ and ${ }^{\psi} \mathfrak{D}^{\sharp}$, the result of the multiplication is an operator acting on $\tilde{S}$-valued (not on $V$-valued) functions.

Finally we can "multiply", in the sense of the Hurwitz multiplication, operators of the type of $\mathcal{D}_{\psi}$ :

$$
\mathcal{D}_{\psi} \circ \mathcal{D}_{\psi}: f \mapsto \mathcal{D}_{\psi} \circ\left(\mathcal{D}_{\psi} \circ f\right)=\mathcal{D}_{\psi}\left[\mathcal{D}_{\psi}[f]\right] .
$$

The operator $\mathcal{D}_{\psi} \circ \mathcal{D}_{\psi}$ acts on $V$-valued functions and in this sense $\mathcal{D}_{\psi} * \mathcal{D}_{\psi}^{\sharp} \neq{ }^{\psi} \mathfrak{D} *{ }^{\psi} \mathfrak{D}^{\sharp}$.

2.6. For a fixed set $\psi$ introduce the differential $\ell$-form

$$
\sigma_{\psi, x}^{(\ell)}:=\sum_{\alpha=0}^{\ell}(-1)^{\alpha} \cdot \psi^{\alpha} d x_{[\alpha]},
$$

where $d x_{[\alpha]}$ is the differential $\ell$-form $d x:=d x_{0} \wedge \ldots \wedge d x_{\ell}$ with $d x_{\alpha}$ omitted. The operator of exterior differentiation $d$ acts on such $\tilde{S}$-valued differential forms as a $\tilde{S}$-linear mapping. Then, if $g \in \mathcal{C}^{1}(\Omega, \tilde{S}), f \in \mathcal{C}^{1}(\Omega, V)$, an easy calculation gives

$$
d\left(g * \sigma_{\psi, x}^{(\ell)} \circ f\right)=\left(\mathfrak{D}^{\psi}[g] \circ f+g * \mathcal{D}_{\psi}[f]\right) d x .
$$


and analogously, for $g, h \in \mathcal{C}^{1}(\Omega, \tilde{S})$

$$
d\left(g * \sigma_{\psi, x}^{(\ell)} * h\right)=\left(\mathfrak{D}^{\psi}[g] * h+g * \psi \mathfrak{D}[h]\right) d x .
$$

2.7. If now we assume that $\Omega$ is a bounded domain with smooth enough boundary $\Gamma=\partial \Omega$, then, application of the Stokes formula immediately gives the following equalities:

$$
\begin{aligned}
& \int_{\Gamma} g * \sigma_{\psi, x}^{(\ell)} \circ f=\int_{\Omega}\left(\mathfrak{D}^{\psi}[g] \circ f+g * \mathcal{D}_{\psi}[f]\right) d x, \\
& \int_{\Gamma} g * \sigma_{\psi, x}^{(\ell)} * h=\int_{\Omega}\left(\mathfrak{D}^{\psi}[g] * h+g *{ }^{\psi} \mathfrak{D}[h]\right) d x
\end{aligned}
$$

2.8. Up to now we assumed no restrictions on $\eta$. But if we want to have a good function theory, we should limit ourselves to the cases where $\eta=I_{p+1}$ or $\eta=\left(\begin{array}{cc}1 & 0 \\ 0 & -I_{p}\end{array}\right)$. Let one of these conditions be fulfilled. Denote by $\Delta_{\ell+1}(\tilde{S})$ and $\Delta_{\ell+1}(V)$ the usual $(\ell+1)$ dimensional Laplace operator acting on $\mathcal{C}^{2}(\Omega, \tilde{S})$ and $\mathcal{C}^{2}(\Omega, V)$, respectively.

Then

$$
\begin{aligned}
\psi_{\mathfrak{D}^{\sharp} *} * \mathfrak{D}={ }^{\psi} \mathcal{D} *{ }^{\psi} \mathfrak{D}^{\sharp} & =\mathfrak{D}^{\psi \sharp} * \mathfrak{D}^{\psi}=\mathfrak{D}^{\psi} * \mathfrak{D}^{\psi \sharp}=\Delta_{\ell+1}(\tilde{S}), \\
\mathcal{D}_{\psi} * \mathcal{D}_{\psi}^{\sharp} & =\mathcal{D}_{\psi}^{\sharp} * \mathcal{D}_{\psi}=\Delta_{\ell+1}(V) .
\end{aligned}
$$

It is easy to describe all $\psi$ 's with the properties (2.11)-(2.12).

2.9. It is well-known that most part of the usual one-dimensional complex analysis (i.e. the theory of holomorphic functions of one complex variable) can be constructed starting from only two facts: a) factorization of the Laplace operator by the conjugate Cauchy-Riemann operators and b) the Green's (or 2-dimensional Stokes) formula. Some multidimensional generalizations, such as the quaternionic and the Clifford analysis, are based on these two facts (one can find the detailed substantiation of this point of view in $[\mathrm{S}]$, for example).

Formulas (2.9) and (2.11), as well as (2.10) and (2.12) show that we can develop the corresponding theories for $V$-valued and $\tilde{S}$-valued functions in the same way. We will show the initial part of this procedure just to illustrate the idea. We shall use the notations

$$
\operatorname{ker} \mathcal{D}_{\psi}=: \mathfrak{N}_{\psi}(\Omega, V) ; \quad \operatorname{ker}^{\psi} \mathfrak{D}=:{ }^{\psi} \mathfrak{M}(\Omega, \tilde{S}) ; \quad \operatorname{ker} \mathfrak{D}^{\psi}=: \mathfrak{M}^{\psi}(\Omega, \tilde{S})
$$

and call the elements of these sets $V$-valued and $\tilde{S}$-valued hyperholomorphic functions, respectively (in the latter case adding sometimes the word "left" or "right").

2.10. Let $\theta_{\ell+1}$ denote the fundamental solution of $\Delta_{\ell+1}$ in $\mathbb{R}^{\ell+1}$, i.e. $\Delta_{\ell+1}\left(\theta_{\ell+1}\right)=\delta$,

$$
\theta_{\ell+1}: x \in \mathbb{R}^{\ell+1} \backslash\{0\} \rightarrow \frac{1}{(1-\ell)\left|\mathbb{S}^{\ell}\right|} \cdot|x|^{1-\ell},
$$

where $\left|\mathbb{S}^{\ell}\right|$ is the area of the unit sphere in $\mathbb{R}^{\ell+1}$. We cannot identify $\theta_{\ell+1}$ in a natural way with a $V$-valued function, but we can identify it with the $\tilde{S}$-valued function $\theta_{\ell+1} \cdot \epsilon_{0}$. Hence we can introduce the function

$$
\begin{aligned}
\mathcal{K}_{\psi}(x) & :={ }^{\psi} \mathfrak{D}^{\sharp}\left[\theta_{\ell+1}\right](x)=\mathfrak{D}^{\psi \sharp}\left[\theta_{\ell+1}\right](x)= \\
& =\frac{1}{\left|\mathbb{S}^{\ell}\right| \cdot|x|^{\ell+1}} \cdot \sum_{\alpha=0}^{\ell} \psi^{\alpha \sharp} \cdot x_{\alpha},
\end{aligned}
$$


which will play the role of the Cauchy kernel for both theories. It has the following important properties:

a) $\mathcal{K}_{\psi} \in \mathcal{C}^{\infty}\left(\mathbb{R}^{\ell+1} \backslash\{0\}, \tilde{S}\right)$,

b) $\mathcal{K}_{\psi} \in{ }^{\psi} \mathfrak{M}\left(\mathbb{R}^{\ell+1} \backslash\{0\}, \tilde{S}\right) \cap \mathfrak{M}^{\psi}\left(\mathbb{R}^{\ell+1} \backslash\{0\}, \tilde{S}\right)$,

c) Let $y \in \mathbb{R}^{\ell+1}, \mathcal{K}_{\psi, y}(x):=\mathcal{K}_{\psi}(y-x)$; then $\mathcal{K}_{\psi, y} \in{ }^{\psi} \mathfrak{M}\left(\mathbb{R}^{\ell+1} \backslash\{y\} \cap \mathfrak{M}^{\psi}\left(\mathbb{R}^{\ell+1} \backslash\{y\}\right)\right.$.

2.11. Theorem (Borel-Pompeiu formula). Let $f \in \mathcal{C}^{1}(\bar{\Omega}, \tilde{S})$, then for $\forall x \in \Omega$,

$$
f(x)=\int_{\Gamma} \mathcal{K}_{\psi}(r-x) * \sigma_{\psi, r}^{(\ell)} \circ f(r)-\int_{\Omega} \mathcal{K}_{\psi}(r-x) * \mathcal{D}_{\psi}[f](r) d r
$$

and

$$
g(x)=\int_{\Gamma} \mathcal{K}_{\psi}(r-x) * \sigma_{\psi, r}^{(\ell)} * g(r)-\int_{\Omega} \mathcal{K}_{\psi}(r-x) *{ }^{\psi} \mathfrak{D}[g](r) d r
$$

i.e.

$$
g(x)=\int_{\Gamma} g(r) * \sigma_{\psi, r}^{(\ell)} * \mathcal{K}_{\psi}(r-x)-\int_{\Omega} \mathfrak{D}_{\psi}[g] * \mathcal{K}_{\psi}(r-x) d r
$$

Proof. Cut out a small ball centered in $x$, apply (2.9) and (2.10) to the rest of $\Omega$; substitute $\mathcal{K}_{\psi}$ instead of $g$ or $f$. Standard routine calculations give the answer.

2.12. TheOREM (Cauchy integral formula). Let $f \in \mathfrak{N}_{\psi}(\bar{\Omega}, V), g \in \mathfrak{M}^{\psi}(\bar{\Omega}, \tilde{S}), h \in$ ${ }^{\psi} \mathfrak{M}(\bar{\Omega}, \tilde{S})$, then for $\forall x \in \Omega$

$$
\begin{aligned}
f(x) & =\int_{\Gamma} \mathcal{K}_{\psi}(r-x) * \sigma_{\psi, r}^{(\ell)} \circ f(r), \\
h(x) & =\int_{\Gamma} \mathcal{K}_{\psi}(r-x) * \sigma_{\psi, r}^{(\ell)} \circ h(r), \\
g(x) & =\int_{\Gamma} g(r) * \sigma_{\psi, r}^{(\ell)} \mathcal{K}_{\psi}(r-x) .
\end{aligned}
$$

Proof. Directly from Theorem 2.11 .

\section{Derivation of Hurwitz analysis from Clifford analysis}

3.1. We start again, as in 2.1 , from a Hurwitz pair $(S, V, \circ)$, an arbitrary integer $\ell: 2 \leq \ell \leq p$, and a domain $\Omega$ in $\mathbb{R}^{\ell+1}$. To simplify our description, we will consider only the case $\psi=\psi_{s t}=\left(\epsilon_{0}, \epsilon_{1}, \ldots, \epsilon_{m}\right)$ and we will write $\mathcal{D}_{\psi_{s t}}=: \mathcal{D}_{s t}=: \mathcal{D},{ }^{\psi_{s t}} \mathfrak{D}=:{ }^{s t} \mathfrak{D}$ and so on.

3.2. Let $C l_{r, s}$ and $\pi_{n}$ denote the Clifford algebra and its irreducible representation, respectively. $\pi_{n}$ acts on elements of $C l_{r, s}$ but we can consider it to act on $C l_{r, s}$-valued functions: $\pi_{n}[f](x):=\pi_{n}[f(x)]$. We will use the same notation $\pi_{n}$ for this new mapping, and we will keep this agreement for other mappings from the diagram in 1.12. 
3.3. The operators ${ }_{s t} \mathbb{D}$ and $\mathbb{D}_{s t}$, together with their conjugates ${ }_{s t} \overline{\mathbb{D}}$ and $\overline{\mathbb{D}}_{s t}$, are defined on $\mathcal{C}^{1}\left(\Omega, C l_{r, s}\right)$ by

$$
\begin{aligned}
& { }_{s t} \mathbb{D}[f]:=\sum_{k=0}^{\ell} i_{k} \cdot \frac{\partial f}{\partial x_{k}}, \\
& \mathbb{D}_{s t}[f]:=\sum_{k=0}^{\ell} \frac{\partial f}{\partial x_{k}} \cdot i_{k}, \\
& s t \overline{\mathbb{D}}[f]:=\sum_{k=0}^{\ell} \overline{i_{k}} \cdot \frac{\partial f}{\partial x_{k}}, \\
& \overline{\mathbb{D}}_{s t}[f]:=\sum_{k=0}^{\ell} \frac{\partial f}{\partial x_{k}} \cdot \overline{i_{k}} .
\end{aligned}
$$

The following facts are well-known (see, for instance, [BDS])

1. Let $\omega_{s t, x}^{(\ell)}:=\sum_{k=0}^{\ell}(-1)^{k} \cdot i_{k} d x_{[k]}\left(\right.$ cf. 2.6) and let $f, g \in \mathcal{C}^{1}\left(\bar{\Omega}, C l_{r, s}\right)$. Then

$$
d\left(g \cdot \omega_{s t, x}^{(\ell)} \cdot f\right)=\left(\mathbb{D}_{s t}[g](x) \cdot f(x)+g \cdot{ }_{s t} \mathbb{D}[f](x)\right) d x .
$$

2. Under the same assumptions

$$
\int_{\Gamma} g(x) \cdot \omega_{s t, x}^{(\ell)} \cdot f(x)=\int_{\Omega}\left\{\mathbb{D}_{s t}[g](x) \cdot f(x)+g(x) \cdot s t \mathbb{D}[f](x)\right\} d x .
$$

3. Assume now that $r=0, s=p$. Then

$$
{ }_{s t} \mathbb{D} \cdot{ }_{s t} \overline{\mathbb{D}}={ }_{s t} \overline{\mathbb{D}} \cdot{ }_{s t} \mathbb{D}=\Delta_{\ell+1} \text {. }
$$

4. Functions from $\operatorname{ker}_{s t} \mathbb{D}=\mathcal{P}\left(\Omega ; C l_{0, p}\right)$ are called (left) $C l_{0, p}$-valued hyperholomorphic functions. Similarly for $\mathbb{D}_{\text {st }}$.

5. If $\theta_{\ell+1}$ is defined as in (2.14), then the function

$$
K(x):={ }_{s t} \mathbb{D}\left[\theta_{\ell+1}\right](x)=\mathbb{D}_{s t}\left[\theta_{\ell+1}\right](x)=\frac{1}{\left|\mathbb{S}^{\ell}\right| \cdot|x|^{\ell+1}} \sum_{k=0}^{\ell} \overline{i_{k}} \cdot x_{k}
$$

is the analog of the Cauchy kernel for this function theory.

6. The Borel-Pompeiu formula is true:

$$
\begin{gathered}
f(x)=\int_{\Gamma} K(r-x) \cdot \omega_{s t, x}^{(\ell)} \cdot f(x)-\int_{\Omega} K(r-x) \cdot s t \mathbb{D}[f](x) d x= \\
\int_{\Gamma} f(x) \cdot \omega_{s t, x}^{(\ell)} \cdot K(r-x)-\int_{\Omega} \mathbb{D}_{s t}[f](x) \cdot K(r-x) \cdot d x .
\end{gathered}
$$

7. If $g$ is right-hyperholomorphic and $f$ is left-hyperholomorphic, then

$$
\int_{\Gamma} g(x) \cdot \omega_{s t, x}^{(\ell)} \cdot f(x)=0 .
$$

8. The Cauchy integral formula holds: for a left-hyperholomorphic function,

$$
f(x)=\int_{\Gamma} K(r-x) \cdot \omega_{s t, x}^{(\ell)} \cdot f(x)
$$

and analogously for a right-hyperholomorphic function. 
3.4. Applying 1.13 we have:

$$
\begin{aligned}
& \tilde{\pi}\left({ }_{s t} \mathbb{D}[f]\right)=\tilde{\pi}\left({ }_{s t} \mathbb{D} \cdot f\right)= \\
= & \sum_{k=0}^{\ell} \tilde{\pi}\left(i_{k} \cdot \frac{\partial}{\partial x_{k}}\right) * \tilde{\pi}(f)= \\
= & \sum_{k=0}^{\ell} \epsilon_{k} \cdot \frac{\partial}{\partial x_{k}} * \tilde{f}={ }^{s t} \mathfrak{D}[\tilde{f}]
\end{aligned}
$$

for any $\tilde{S}$-valued function $\tilde{f}$. Hence

$$
\tilde{\pi}\left({ }_{s t} \mathbb{D}\right)={ }^{s t} \mathfrak{D} .
$$

Analogously

$$
\tilde{\pi}\left(\mathbb{D}_{s t}\right)={ }^{s t} \mathfrak{D} .
$$

Again as in 1.13 we obtain:

$$
\begin{aligned}
P \gamma \tilde{\pi}(s t \mathbb{D}[f]) & =\sum_{k=0}^{\ell} \pi_{n}\left(i_{k} \cdot \frac{\partial}{\partial x_{k}}\right) \circ \operatorname{P\gamma } \tilde{\pi}(f)= \\
& =\sum_{k=0}^{\ell} \epsilon_{k} \frac{\partial}{\partial x_{k}} \circ \tilde{f}=\mathcal{D}[\tilde{f}]
\end{aligned}
$$

for any $V$-valued function $\tilde{f}$. Hence

$$
\operatorname{P\gamma \pi }\left({ }_{s t} \mathbb{D}\right)=\mathcal{D} .
$$

Easy calculations give also:

$$
\begin{aligned}
\tilde{\pi}\left({ }_{s t} \overline{\mathbb{D}}\right) & ={ }^{s t} \mathfrak{D}^{\sharp}, \\
\tilde{\pi}\left(\overline{\mathbb{D}}_{s t}\right) & =\mathfrak{D}^{s t \sharp}, \\
P U \pi(s t & =\mathfrak{D}^{\sharp}, \\
\tilde{\mathbb{D}}) & \left(\omega_{s t}^{(\ell)}\right)=\sigma_{s t}^{(\ell)} .
\end{aligned}
$$

3.5. Now we can obtain all results of Section 2 as direct corollaries of the corresponding facts from Clifford analysis. Let us show this on Theorem 2.13. Taking formula (3.1) and applying the technique from 3.1-3.4 we have:

$$
\begin{gathered}
g(x)=\tilde{\pi}(\tilde{g})(x)=\int_{\Gamma} \tilde{\pi}\left(K(r-x) \cdot \omega_{s t, x}^{(\ell)} \cdot \tilde{g}(x)\right)= \\
=\int_{\Gamma} \tilde{\pi}(K(r-x)) * \tilde{\pi}\left(\omega_{s t, x}^{(\ell)}\right) * \tilde{\pi}(\tilde{g}(s))= \\
=\int_{\Gamma} K(r-x) * \sigma_{s t, x}^{(\ell)} g(x),
\end{gathered}
$$

which coincides with the corresponding equality in Theorem 2.12. 


\section{References}

[BDS] F. Brackx, R. Delanghe, F. Sommen, Clifford Analysis, Pitman Research Notes in Math. 76, 1982.

[H1] A. Hurwitz, Über die Komposition der quadratischen Formen von beliebig vielen Variablen, Nachrichten von der Königlichen Gesellschaft der Wiessenschaften zu Göttingen Math. phys. Kl. (1898), 308-316, reprinted in: A. Hurwitz, Mathematische Werke II, Birkhäuser Verlag, Basel, 1933, 565-571.

[H2] —, Über die Komposition der quadratischen Formen, Math. Ann. 88 (1923), 1-25, reprinted in: A. Hurwitz, Mathematische Werke II, Birkhäuser Verlag, Basel, 1933, 641-666.

[KR1] W. Królikowski and E. Ramírez de Arellano, Fueter-Hurwitz regular mappings and an integral representation, in: Clifford Algebras and Their Applications in Mathematical Physics, A. Micali et al., eds., Kluwer Acad. Publishers, Dordrecht 1992, $221-237$

[KR2] - - - Polynomial solutions of the Fueter-Hurwitz equation, Cont. Math. 137 (1992), 297-305.

[LR] J. Ławrynowicz and J. Rembieliński, Pseudo-euclidean Hurwitz pairs and generalized Fueter equations, in: Clifford Algebras and Their Applications in Mathematical Physics, J.S.R. Chisholm and A.K. Common, eds., D. Reidel Publ. Co., Dordrecht 1986, 39-48.

[RSV1] E. Ramírez de Arellano, M. V. Shapiro, N. L. Vasilevski, A canonical relation between Hurwitz pairs and Clifford algebras, Reporte Interno No. 112, Dep. Mat., CINVESTAV del IPN., Mexico City, 1993, 34 pp.

[RSV2] - - - Hurwitz pairs and Clifford algebra representations, in: Clifford Algebras and Their Applications in Mathematical Physics, F. Brackx, R. Delanghe and H. Serras eds., Kluwer Acad. Publishers, Dordrecht 1993, 171-181.

[S] M. V. Shapiro, Some remarks on generalizations of the one-dimensional complex analysis: hypercomplex approach, Reporte Interno No. 131, Dep. Mat., CINVESTAV del IPN., Mexico City, 1993, 23 pp.

[Sh] D. B. Shapiro, Products of sums of squares, Expo. Math. 2 (1984), 235-261.

[SY] T. L. Smith and P. Yiu, Construction of sums of squares formulae with integer coefficients, Bol. Soc. Mat. Mexicana (2) 37 (1992), 479-495. 